\title{
Optimal evaluations for the bias of trimmed means of $k$ th record values
}

\author{
Mariusz Bieniek
}

Received: 10 January 2014 / Published online: 27 September 2014

(C) The Author(s) 2014. This article is published with open access at Springerlink.com

\begin{abstract}
We provide sharp upper and lower mean-variance bounds on the expectations of trimmed means of $k$ th record values from general family of distributions. Also we improve these bounds in the case of non-trimmed means for parent distributions with decreasing density or decreasing failure rate. They can be viewed as bounds on the bias of approximation of expectation of the parent population by mean or trimmed mean of record values. The results are illustrated with numerical examples.
\end{abstract}

Keywords Sharp bounds $\cdot$ Schwarz inequality $\cdot$ Trimmed mean $\cdot k$ th record values Decreasing density $\cdot$ Decreasing failure rate

Mathematics Subject Classification $\quad 62 \mathrm{G} 30 \cdot 60 \mathrm{E} 15$

\section{Introduction}

Let $\left\{X_{n}, n \geq 1\right\}$ be a sequence of i.i.d. random variables with continuous common cummulative distribution function $F$ with finite mean

$$
\mu=\int_{0}^{1} F^{-1}(u) \mathrm{d} u
$$

and finite variance

$$
\sigma^{2}=\int_{0}^{1}\left(F^{-1}(u)-\mu\right)^{2} \mathrm{~d} u .
$$

\footnotetext{
M. Bieniek ( $\square)$

University of Maria Curie-Skłodowska, Pl. M. Curie-Skłodowskiej 1, 20-031 Lublin, Poland e-mail: mariusz.bieniek@umcs.lublin.pl
} 
Let $\left\{Y_{n}^{(k)}, n \geq 1\right\}$ denote the sequence of $k$ th record values of $\left\{X_{n}, n \geq 1\right\}$, defined by Dziubdziela and Kopociński (1976) as $Y_{n}^{(k)}=X_{U_{k}(n): U_{k}(n)+k-1}$, where $U_{k}(1)=1$ and

$$
U_{k}(n+1)=\min \left\{j>U_{k}(n): X_{j: j+k-1}>X_{U_{k}(n): U_{k}(n)+k-1}\right\}, \quad n \geq 1 .
$$

For $1 \leq r \leq n$ let

$$
T_{r, n}^{(k)}=\frac{1}{n-r+1} \sum_{i=r}^{n} Y_{i}^{(k)},
$$

denote the trimmed mean of $k$ th record values and in particular for $n \geq 2$

$$
S_{n}^{(k)}=T_{1, n}^{(k)}=\frac{1}{n} \sum_{i=1}^{n} Y_{i}^{(k)}
$$

denotes the mean of $n$ first $k$ th record values. Assume that we are given not the observations $X_{1}, X_{2}, \ldots$, but only $k$ th record values and we want to estimate the mean $\mu$ of the parent distribution $F$. To this aim we propose the usage of $T_{r, n}^{(k)}$ and $S_{n}^{(k)}$. The approximation of $\mu$ by $T_{r, n}^{(k)}$ makes sense for $n \leq k$, since then there is a chance that some records $Y_{i}^{(k)}, r \leq i \leq n$, have expected values less than $\mu$. Other values of $n$ and $k$ (i.e. $n>k$ ) are studied for completeness.

In order to study the quality of approximation of $\mu$ by $T_{r, n}^{(k)}$ we evaluate the bias of $T_{r, n}^{(k)}$ expressed in standard deviation units. In other words we seek for sharp lower and upper bounds on

$$
\frac{E T_{r, n}^{(k)}-\mu}{\sigma}
$$

for general $F$ with finite $\sigma$. The mean $S_{n}^{(k)}$ seems to be most reasonable estimator of $\mu$ among $T_{r, n}^{(k)}, 1 \leq r \leq n$, but as we show that left trimming improves lower bounds so it also makes sense to study $T_{r, n}^{(k)}$ with $r>1$ as well. The first idea is to note that by obvious inequalities

$$
Y_{r}^{(k)} \leq T_{r, n}^{(k)} \leq Y_{n}^{(k)}
$$

it may suffice to apply upper bounds on $E Y_{n}^{(k)}$ of Raqab (1997) or Raqab and Rychlik (2002), and lower bounds on $E Y_{r}^{(k)}$ of Goroncy and Rychlik (2011). But the bounds obtained this way are not optimal since the equality in the above inequality holds iff $Y_{r}^{(k)}=Y_{n}^{(k)}$ which holds with probability 0 .

From numerical computations it appears that the smallest upper error is commited if $r=1$. Therefore we improve upper bounds on

$$
\frac{E S_{n}^{(k)}-\mu}{\sigma}
$$

for $F$ coming from restricted families of distributions with decreasing density (DD) and with decreasing failure rate (DFR) defined by convex ordering of distributions. 
For any distribution function $F$ its quantile function is defined as

$$
F^{-1}(u)=\sup \{x \in \mathbb{R}: F(x) \leq u\}, \quad u \in(0,1) .
$$

For two distribution functions $F$ and $G$ we write $F \succ_{c} G$ if and only iff $F^{-1} G$ is convex function on the support of $G$. Then we write $F \in \mathrm{DD}$ if $F \succ_{c} U$, where $U$ is the cdf of the uniform distribution on $[0,1]$, and $F \in$ DFR if $F \succ_{c} V$, where $V(x)=1-\mathrm{e}^{-x}, x \geq 0$, is the cdf of the standard exponential distribution.

The bounds are derived by applications of the projection method. For its full explaination and numerous examples the reader is referred to the monograph of Rychlik (2001). The bounds valid in the general class of distributions are obtained by Moriguti's approach of the greatest convex minorants and the bounds in restricted classes - by making use of slight generalization of the results of Danielak (2003). For exhaustive review on results concerning bounds on expectations of $k$ th record values and their functions valid in general and restricted families of distributions we refer to Section 1 of Goroncy and Rychlik (2011).

In Sect. 2 we recall necessary results on projection method. The key step in application of these results is to determine the shapes of the functions to be projected. This is done in Sect. 3 with the aid of the variation diminishing property of the densities of $k$ th record values from uniform distribution. This property is stated and proved by Gajek and Okolewski (2003), but here we give its new simple proof based on the Descartes' rule of signs. Section 4 contains the main results on bounds on $E T_{r, n}^{(k)}$ for general distributions. In Sect. 5 we focus on restricted families of distributions. First in Sect. 5.1 we need to generalize the results of Danielak (2003) where the projected functions $h$ satisfy $h(0)=0$ while in our case we have $h(0)>0$. The solution to this new problem is similar but it cannot be simply inferred from the results of Danielak (2003). Then in Sects. 5.2 and 5.3 we present the main results on bounds on $E S_{n}^{(k)}$ for DD and DFR distributions. Finally in Sect. 6 we present numerical computations of the bounds obtained in this paper.

\section{Auxiliary results on the projection method}

Using the representation (valid only for continuous $F$ )

$$
E Y_{n}^{(k)}=\int_{0}^{1} F^{-1}(u) f_{n}^{(k)}(u) \mathrm{d} u
$$

where

$$
f_{n}^{(k)}(u)=\frac{k^{n}}{(n-1) !}(1-u)^{k-1}(-\log (1-u))^{n-1}, \quad u \in(0,1),
$$

denotes the density of $k$ th record value from uniform $U(0,1)$ distribution, we easily get

$$
E T_{r, n}^{(k)}=\int_{0}^{1} F^{-1}(u) g_{r, n}^{(k)}(u) \mathrm{d} u
$$


where

$$
g_{r, n}^{(k)}(u)=\frac{1}{n-r+1} \sum_{i=r}^{n} f_{i}^{(k)}(u), \quad u \in(0,1) .
$$

Since $g_{r, n}^{(k)}$ integrates to 1 , and $F^{-1}-\mu$ integrates to 0 we get

$$
E T_{r, n}^{(k)}-\mu=\int_{0}^{1}\left(F^{-1}(u)-\mu\right)\left(g_{r, n}^{(k)}(u)-1\right) \mathrm{d} u .
$$

By the projection method and Schwarz inequality

$$
\frac{E T_{r, n}^{(k)}-\mu}{\sigma} \leq\left\|\bar{g}_{r, n}^{(k)}-1\right\|_{2}
$$

where $\bar{g}_{r, n}^{(k)}$ denotes the projection of $g_{r, n}^{(k)}$ onto the convex cone $\mathscr{C}$ of nondecreasing functions on $[0,1]$. The bound is attained if

$$
\frac{F^{-1}(u)-\mu}{\sigma}=\frac{\bar{g}_{r, n}^{(k)}(u)-1}{\left\|\bar{g}_{r, n}^{(k)}-1\right\|_{2}} .
$$

To derive lower bounds Danielak and Rychlik (2003) used the symmetry of distributions of order statistics, but this symmetry no longer holds for record values. So the lower bound is derived analogously to the upper one noting that

$$
-\left(E T_{r, n}^{(k)}-\mu\right) \leq \sigma\left\|\overline{-g}_{r, n}^{(k)}+1\right\|_{2},
$$

where $\overline{-g}_{r, n}^{(k)}$ denotes the projection of $-g_{r, n}^{(k)}$ onto $\mathscr{C}$. The equality is attained if

$$
\frac{F^{-1}(u)-\mu}{\sigma}=\frac{\overline{-g}_{r, n}^{(k)}(u)+1}{\left\|\overline{-g}_{r, n}^{(k)}+1\right\|_{2}} .
$$

This gives

$$
-\left\|\overline{-g}_{r, n}^{(k)}+1\right\|_{2} \leq \frac{E T_{r, n}^{(k)}-\mu}{\sigma} \leq\left\|\bar{g}_{r, n}^{(k)}-1\right\|_{2} .
$$

Therefore it suffices to determine the projections of $g_{r, n}^{(k)}$ and $-g_{r, n}^{(k)}$ onto $\mathscr{C}$.

By Theorem 1 of Moriguti (1953), for any function $g:[0,1] \rightarrow \mathbb{R}$ its projection $\bar{g}$ onto $\mathscr{C}$ is the right-hand derivative of the greatest convex minorant $\bar{G}$ of the antiderivative

$$
G(x)=\int_{0}^{x} g(t) \mathrm{d} t
$$

of $g$. In our case $g$ is either $g_{r, n}^{(k)}$ or $-g_{r, n}^{(k)}$, so to determine convexity regions of their antiderivatives, we need to determine monotonicity properties of $g_{r, n}^{(k)}$. This is done in Sect. 3 and the projections $\bar{g}_{r, n}^{(k)}$ and $\overline{-g}_{r, n}^{(k)}$ are determined in Sect. 4. 
In Sect. 5 we apply the projection method in a more subtle way. We consider the Hilbert space $\mathscr{L}_{W}^{2}$, where $W:[a, d) \mapsto \mathbb{R}$ is a fixed absolutely continuous distribution function, of real functions on $[a, d)$ square integrable with weight $w=W^{\prime}$ such that

$$
\int_{a}^{d} x^{2} w(x) \mathrm{d} x<\infty
$$

Let $\|g\|_{W}=\left(\int_{a}^{d}(g(x))^{2} w(x) \mathrm{d} x\right)^{1 / 2}$ denote the norm in $\mathscr{L}_{W}^{2}$. Then

$$
\mathscr{C}_{W}=\left\{g \in \mathscr{L}_{W}^{2}: g \text { is nondecreasing and convex }\right\}
$$

is a convex cone in $\mathscr{L}_{W}^{2}$. Let $P_{W}: \mathscr{L}_{W}^{2} \mapsto \mathscr{C}_{W}$ denote the projection operator onto $\mathscr{C}_{W}$. Now writting $h_{n}^{(k)}=g_{1, n}^{(k)}$ and

$$
\begin{aligned}
E S_{n}^{(k)}-\mu & =\int_{0}^{1}\left(F^{-1}(u)-\mu\right)\left(h_{n}^{(k)}(u)-1\right) \mathrm{d} u \\
& =\int_{a}^{d}\left(F^{-1} W(u)-\mu\right)\left(h_{n}^{(k)} W(u)-1\right) w(u) \mathrm{d} u,
\end{aligned}
$$

we get by the projection method and Schwarz' inequality

$$
\frac{E S_{n}^{(k)}-\mu}{\sigma} \leq C_{W}(n, k):=\left(\left\|P_{W} \hat{h}_{n}^{(k)}\right\|_{W}^{2}-1\right)^{1 / 2},
$$

where $\hat{h}_{n}^{(k)}=h_{n}^{(k)} W$ and $P_{W} \hat{h}_{n}^{(k)}$ is the projection of $\hat{h}_{n}^{(k)}$ onto the convex cone $\mathscr{C}_{W}$. This bound is sharp and the equality in (4) is attained if

$$
\frac{F^{-1} W(x)-\mu}{\sigma}=\frac{P_{W} \hat{h}_{n}^{(k)}(x)-1}{C_{W}(n, k)} .
$$

Since this time we approximate the functions $\hat{h}_{n}^{(k)}$ by convex functions we need to study their convexity properties. This is also done in Sect. 3 and the projection $P_{W} \hat{h}_{n}^{(k)}$ is determined in Sect. 5.1.

\section{Shapes of projected functions}

To determine the exact shape of functions $g_{r, n}^{(k)}$ and $h_{n}^{(k)}$ it suffices to study the sign changes of their first and second derivatives. Since $g_{r, n}^{(k)}$ and $h_{n}^{(k)}$ are linear combinations of $f_{1}^{(k)}, \ldots, f_{n}^{(k)}$ their differentiation is easy, but studying sign changes of their derivatives is not easy at all. But as we show now, the derivatives are also linear combinations of $f_{1}^{(k)}, \ldots, f_{n}^{(k)}$. 
Namely, to obtain first and second derivatives of $g_{r, n}^{(k)}$ we use the identities

$$
\left(f_{n}^{(k)}\right)^{\prime}(x)=\frac{1}{1-x}\left(k f_{n-1}^{(k)}(x)-(k-1) f_{n}^{(k)}(x)\right),
$$

and

$\left(f_{n}^{(k)}\right)^{\prime \prime}(x)=\frac{1}{(1-x)^{2}}\left(k^{2} f_{n-2}^{(k)}(x)-k(2 k-3) f_{n-1}^{(k)}(x)+(k-1)(k-2) f_{n}^{(k)}(x)\right)$,

which may be easily verified by direct computations. They hold true for $n=1$ and $n=2$ as well if we adopt the convention $f_{-1}^{(k)}=f_{0}^{(k)} \equiv 0$. Therefore we easily get

$$
\left(g_{r, n}^{(k)}\right)^{\prime}(x)=\frac{1}{(n-r+1)(1-x)} \sum_{i=r-1}^{n} a_{i} f_{i}^{(k)}(x),
$$

where

$$
\begin{aligned}
a_{r-1} & =k, \\
a_{i} & =1, \quad r \leq i<n, \\
a_{n} & =-(k-1) .
\end{aligned}
$$

Moreover,

$$
\left(g_{r, n}^{(k)}\right)^{\prime \prime}(x)=\frac{1}{(n-r+1)(1-x)^{2}} \sum_{i=r-2}^{n} b_{i} f_{i}^{(k)}(x),
$$

where

$$
\begin{aligned}
b_{r-2} & =k^{2}, \\
b_{r-1} & =-k(k-3), \\
b_{i} & =2, \quad r \leq i \leq n-2, \\
b_{n-1} & =-k^{2}+2, \\
b_{n} & =(k-1)(k-2) .
\end{aligned}
$$

The sign changes of such combinations can be studied by variation diminishing property (VDP) of $f_{1}^{(k)}, \ldots, f_{n}^{(k)}$ of Gajek and Okolewski (2003).

Theorem 1 The number of zeros of any linear combination $\sum_{i=1}^{n} a_{i} f_{i}^{(k)}$ in $(0,1)$ does not exceed the number of sign changes in the sequence $a_{1}, \ldots, a_{n}$ of its coefficients. Moreover, the first and the last sign of the combination are the same as the signs of the first and the last nonzero coefficients, respectively.

This theorem was proved in Gajek and Okolewski (2003) using the notion of total positivity, but here we give new simpler proof of its first part based on Descartes' rule of signs (see Wang 2004; Komornik 2006, for its elementary proofs). 
Proof Note that

$$
\sum_{i=1}^{n} a_{i} f_{i}^{(k)}(x)=(1-x)^{k-1} P(-\log (1-x)),
$$

where

$$
P(z)=b_{1}+b_{2} z+\cdots+b_{n} z^{n-1},
$$

with $b_{i}=\frac{k^{i}}{(i-1) !} a_{i}, i=1, \ldots, n$. Now, the number of zeros of $\sum_{i=1}^{n} a_{i} f_{i}^{(k)}$ in $(0,1)$ is the same as the number of zeros of the polynomial $P$ in $\mathbb{R}_{+}$. By Descartes' rule of signs this number does not exceed the number of sign changes in $b_{1}, \ldots, b_{n}$, which in turn is equal to the number of sign changes of $a_{1}, \ldots, a_{n}$.

Now carefully analyzing the signs of coefficients in the expansions of first and second derivatives, we can determine the shapes of $g_{r, n}^{(k)}$ and $h_{n}^{(k)}$. First we consider the case $r=1$, where $g_{1, n}^{(k)}=h_{n}^{(k)}$, which will be of special interest to us.

Theorem 2 (a) Let $k=1$. The functions $h_{n}^{(1)}, n \geq 2$, are convex increasing from $1 / n$ to $+\infty$.

(b) Let $k \geq 2$. The functions $h_{n}^{(k)}, n \geq 2$, are increasing from $k / n$, and then decreasing to 0. Moreover:

(i) for $k=2$ the function $h_{2}^{(2)}$ is concave, and $h_{n}^{(2)}, n \geq 3$, are convex-concave.

(ii) for $k \geq 3$ the functions $h_{n}^{(k)}, n \geq 2$, are all convex-concave-convex, with the only exception of $h_{2}^{(3)}$ which is concave-convex.

Now we consider the case when $r \geq 2$.

Theorem 3 (a) Let $k=1$. The functions $g_{r, n}^{(1)}, 2 \leq r<n$, are convex increasing from 0 to $\infty$.

(b) Let $k \geq 2$. The functions $g_{r, n}^{(k)}, 2 \leq r<n$, are increasing-decreasing. Moreover:

(i) for $k=2$ the functions $g_{r, n}^{(2)}, 2 \leq r<n$, are convex-concave.

(ii) for $k=3$ the functions $g_{r, n}^{(3)}, 2 \leq r<n, n \geq 4$, and the functions $g_{r, r+1}^{(k)}$, $k, r \geq 3$, are convex-concave-convex

(iii) for $k \geq 3$ the functions $g_{2,3}^{(k)}$ are concave-convex.

(iv) for $k \geq 4$ the functions $g_{2, n}^{(k)}, n \geq 4$, are either concave-convex or concaveconvex-concave-convex;

(v) for $k \geq 4$ the functions $g_{r, n}^{(k)}, 3 \leq r \leq n-2$, are either convex-concave-convex or convex-concave-convex-concave-convex.

Remark 1 In (b)(iv) and (b)(v) of Theorem 3 both possibilities do occur. For instance numerical computation with Mathematica software show that $g_{2,5}^{(5)}$ is concave-convex and $g_{2,30}^{(10)}$ is concave-convex-concave-convex; and $g_{15,30}^{(10)}$ is convex-concave-convex and $g_{5,30}^{(20)}$ is convex-concave-convex-concave-convex. See Fig. 1 for the graphs of $g_{2,30}^{(10)}$ and $g_{5,30}^{(20)}$.

VDP will be used once more in Sect. 5 to study the behaviour of the functions $K_{W}$ and $L_{W}$ defined in (6) and (7). 

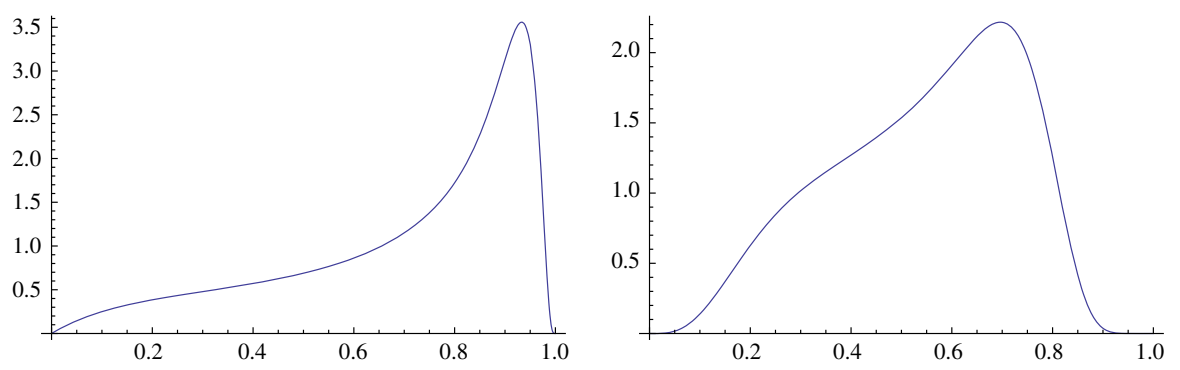

Fig. 1 Exemplary shapes of $g_{2,30}^{(10)}$ (left) and $g_{5,30}^{(20)}$ (right)

\section{General distributions}

The bounds expressed in $\sigma$ units are well known and their derivation is rather standard. Therefore we only sketch the most crucial details.

If $k=1$, by Theorems 2 and 3, the functions $g_{r, n}^{(1)}, 1 \leq r<n$, are increasing and therefore $\bar{g}_{r, n}^{(1)}=g_{r, n}^{(1)}$ and

$$
\frac{E T_{r, n}^{(1)}-\mu}{\sigma_{2}} \leq\left(\int_{0}^{1}\left(g_{1, n}^{(k)}(u)\right)^{2} \mathrm{~d} u-1\right)^{1 / 2} .
$$

On the other hand $-g_{r, n}^{(1)}$ is decreasing and it is obvious that $\overline{-g}_{r, n}^{(1)}=-1$, so

$$
E T_{r, n}^{(1)} \geq \mu
$$

This bound is trivial as it can be derived from obvious inequalities

$$
Y_{n}^{(1)}>\cdots>Y_{1}^{(1)}=X_{1}
$$

and therefore $E Y_{i}^{(1)} \geq \mu$ for all $i=1,2, \ldots$.

If $k \geq 2$, then by Theorems 2 and 3 , the functions $g_{r, n}^{(k)}$ are nonnegative, increasing from 0 , and then decreasing to 0 with the exception of $r=1$ when $g_{1, n}^{(k)}$ starts from $k / n$. Therefore if

$$
G_{r, n}^{(k)}(x)=\int_{0}^{x} g_{r, n}^{(k)}(u) \mathrm{d} u, \quad 0 \leq x \leq 1,
$$

then $G_{r, n}^{(k)}$ is strictly increasing with $G_{r, n}^{(k)}(0)=0$ and $G_{r, n}^{(k)}(1)=1$ and there exists unique $\theta=\theta(k, r, n)$ such that $G_{r, n}^{(k)}$ is convex on $(0, \theta)$ and concave on $(\theta, 1)$.

If $r=1$ and $n \leq k$, then $\left(G_{1, n}^{(k)}\right)^{\prime}(0)=g_{1, n}^{(k)}(0) \geq 1$, and the greatest convex minorant of $G_{1, n}^{(k)}(x)$ is just $x$, so $\bar{g}_{1, n}^{(k)}=1$ and

$$
E T_{1, n}^{(k)} \leq \mu, \quad n \leq k
$$

This bound becomes trivial if we realize that $E Y_{1}^{(k)}=E X_{1: k} \leq E X_{1}=\mu$. 
In all the remaining cases, i.e. $k \geq 2$ and $r=1, n>k$ or $r \geq 2$, there exists unique $\alpha^{*}=\alpha^{*}(k, r, n) \in(0, \theta)$ defined by the equation

$$
1-G_{r, n}^{(k)}\left(\alpha^{*}\right)=\left(1-\alpha^{*}\right) g_{r, n}^{(k)}\left(\alpha^{*}\right),
$$

such that the projection of $g_{r, n}^{(k)}$ onto $\mathscr{C}$ is

$$
\bar{g}_{r, n}^{(k)}(u)= \begin{cases}g_{r, n}^{(k)}(u), & \text { if } 0 \leq u \leq \alpha^{*} \\ g_{r, n}^{(k)}\left(\alpha^{*}\right), & \text { if } \alpha^{*} \leq u \leq 1\end{cases}
$$

On the other hand for $k \geq 2$ and $1 \leq r<n$, there exists unique $\alpha_{*} \in(\theta, 1)$ defined by

$$
G_{r, n}^{(k)}\left(\alpha_{*}\right)=\alpha_{*} g_{r, n}^{(k)}\left(\alpha_{*}\right),
$$

such that

$$
\overline{-g}_{r, n}^{(k)}(u)= \begin{cases}-g_{r, n}^{(k)}\left(\alpha_{*}\right), & \text { if } 0 \leq u \leq \alpha_{*} \\ -g_{r, n}^{(k)}(u), & \text { if } \alpha_{*} \leq u \leq 1\end{cases}
$$

Pluging these functions into (3) and calculating the respective norms we conclude the proof of the following theorem.

Theorem 4 For any continuous $c d f F$ with finite mean $\mu$ and variance $\sigma^{2}$ we have

$$
-\underline{B}_{r, n}^{(k)} \leq \frac{E T_{r, n}^{(k)}-\mu}{\sigma_{2}} \leq \bar{B}_{r, n}^{(k)}, \quad 1 \leq r<n,
$$

where

$$
\left(\bar{B}_{r, n}^{(k)}\right)^{2}= \begin{cases}\int_{0}^{1}\left(g_{r, n}^{(1)}(u)\right)^{2} \mathrm{~d} u-1, & \text { if } k=1, \\ 0, & \text { if } k \geq 2, r=1 \text { and } n \leq k, \\ \int_{0}^{\alpha^{*}}\left(g_{r, n}^{(k)}(u)\right)^{2} \mathrm{~d} u+\left(1-\alpha^{*}\right)\left(g_{r, n}^{(k)}\left(\alpha^{*}\right)\right)^{2}-1, & \text { otherwise. }\end{cases}
$$

and

$$
\left(\underline{B}_{r, n}^{(k)}\right)^{2}= \begin{cases}0, & \text { if } k=1, \\ \alpha_{*}\left(g_{r, n}^{(k)}\left(\alpha_{*}\right)\right)^{2}+\int_{\alpha_{*}}^{1}\left(g_{r, n}^{(k)}(u)\right)^{2} \mathrm{~d} u-1, & \text { if } k \geq 2 .\end{cases}
$$

The bounds are attained in the limit by distributions satisfying (1) for upper bounds, and by (2) for the lower ones. These distributions have analogous form to Raqab and Rychlik (2002) for the upper bounds and Goroncy and Rychlik (2011) for the lower ones and therefore their specification is omitted.

Remark 2 The above theorem could easily be generalized to arbitrary $p \in[1, \infty]$ instead of $p=2$ to provide $p$-norm bounds on the bias of trimmed means of $k$ th record values. 
We close this section with numerical values of the bounds $\bar{B}_{r, n}^{(k)}$ and $\underline{B}_{r, n}^{(k)}$ for $1 \leq$ $r \leq n \leq 10$. In Tables 1, 2 and 3 we present the values of the upper bounds $\bar{B}_{r, n}^{(1)}, \bar{B}_{r, n}^{(2)}$ and $\bar{B}_{r, n}^{(3)}$. Table 4 contains exemplary values of the lower bounds $\underline{B}_{r, n}^{(3)}$. The values in bold correspond to $r=n$, i.e. to single $k$ th record values. They were obtained by Nagaraja (1978) for $k=1$ (Table 1), Raqab (1997) for $k \geq 2$ (Tables 2 and 3) and Goroncy and Rychlik (2011) (Table 4).

In Table 1 we observe that for ordinary record values (i.e. for $k=1$ ) the approximation of $\mu$ by trimmed means is very poor (even for $r=1$ ), but as Tables 2 and 3 show, it significantly improves for $k=2$ or $k=3$. Also in general the upper bounds become smaller if $k$ increases, and they become larger if either $r$ or $n$ increases. The lower bounds become larger as $k$ increases and they decrease with the increase of $r$ or $n$. The above relations reflect elementary inequalities $T_{r, n}^{(k)} \leq T_{r+1, n}^{(k)}$ and $T_{r, n}^{(k)} \leq T_{r, n+1}^{(k)}$ and $T_{r, n}^{(k+1)} \leq T_{r, n}^{(k)}$.

Table 1 The values of $\bar{B}_{r, n}^{(1)}$ for $1 \leq r \leq n \leq 10$

\begin{tabular}{|c|c|c|c|c|c|c|c|c|c|c|}
\hline$r \backslash n$ & 1 & 2 & 3 & 4 & 5 & 6 & 7 & 8 & 9 & 10 \\
\hline 1 & 0. & 0.5 & 1.054 & 1.82 & 3.007 & 4.964 & 8.308 & 14.14 & 24.48 & 42.97 \\
\hline 2 & & 1. & 1.581 & 2.427 & 3.758 & 5.957 & 9.692 & 16.17 & 27.54 & 47.75 \\
\hline 3 & & & 2.236 & 3.24 & 4.796 & 7.323 & 11.56 & 18.82 & 31.45 & 53.7 \\
\hline 4 & & & & 4.359 & 6.245 & 9.232 & 14.13 & 22.39 & 36.58 & 61.3 \\
\hline 5 & & & & & 8.307 & 11.94 & 17.74 & 27.31 & 43.47 & 71.26 \\
\hline 6 & & & & & & 15.84 & 22.89 & 34.22 & 52.95 & 84.61 \\
\hline 7 & & & & & & & 30.38 & 44.11 & 66.27 & 103. \\
\hline 8 & & & & & & & & $\mathbf{5 8 . 5 7}$ & 85.39 & 128.8 \\
\hline 9 & & & & & & & & & 113.4 & 165.9 \\
\hline 10 & & & & & & & & & & 220.5 \\
\hline
\end{tabular}

Table 2 The values of $\bar{B}_{r, n}^{(2)}$ for $1 \leq r \leq n \leq 10$

\begin{tabular}{|c|c|c|c|c|c|c|c|c|c|c|}
\hline$r \backslash n$ & 1 & 2 & 3 & 4 & 5 & 6 & 7 & 8 & 9 & 10 \\
\hline 1 & $\mathbf{0}$. & 0. & 0.154 & 0.353 & 0.562 & 0.787 & 1.038 & 1.326 & 1.663 & 2.065 \\
\hline 2 & & 0.345 & 0.499 & 0.661 & 0.84 & 1.046 & 1.287 & 1.572 & 1.914 & 2.327 \\
\hline 3 & & & 0.736 & 0.912 & 1.1 & 1.312 & 1.558 & 1.851 & 2.204 & 2.634 \\
\hline 4 & & & & 1.145 & 1.356 & 1.588 & 1.853 & 2.164 & 2.537 & 2.989 \\
\hline 5 & & & & & 1.617 & 1.879 & 2.173 & 2.514 & 2.916 & 3.4 \\
\hline 6 & & & & & & 2.191 & 2.524 & 2.903 & 3.346 & 3.873 \\
\hline 7 & & & & & & & 2.91 & 3.339 & 3.833 & 4.414 \\
\hline 8 & & & & & & & & $\mathbf{3 . 8 2 7}$ & 4.384 & 5.032 \\
\hline 9 & & & & & & & & & 5.009 & 5.737 \\
\hline 10 & & & & & & & & & & 6.544 \\
\hline
\end{tabular}


Table 3 The values of $\bar{B}_{r, n}^{(3)}$ for $1 \leq r \leq n \leq 10$

\begin{tabular}{|c|c|c|c|c|c|c|c|c|c|c|}
\hline$r \backslash n$ & 1 & 2 & 3 & 4 & 5 & 6 & 7 & 8 & 9 & 10 \\
\hline 1 & 0. & 0. & 0. & 0.088 & 0.209 & 0.337 & 0.469 & 0.606 & 0.75 & 0.904 \\
\hline 2 & & 0.214 & 0.305 & 0.392 & 0.482 & 0.578 & 0.684 & 0.801 & 0.931 & 1.07 \\
\hline 3 & & & 0.481 & 0.582 & 0.678 & 0.775 & 0.879 & 0.991 & 1.116 & 1.256 \\
\hline 4 & & & & 0.734 & 0.843 & 0.951 & 1.061 & 1.178 & 1.305 & 1.446 \\
\hline 5 & & & & & 0.992 & 1.113 & 1.235 & 1.362 & 1.497 & 1.645 \\
\hline 6 & & & & & & 1.269 & 1.406 & 1.546 & 1.694 & 1.852 \\
\hline 7 & & & & & & & 1.576 & 1.732 & 1.896 & 2.069 \\
\hline 8 & & & & & & & & 1.922 & 2.103 & 2.294 \\
\hline 9 & & & & & & & & & 2.318 & 2.529 \\
\hline 10 & & & & & & & & & & 2.776 \\
\hline
\end{tabular}

Table 4 The values of $\underline{B}_{r, n}^{(3)}$ for $1 \leq r \leq n \leq 10$

\begin{tabular}{|c|c|c|c|c|c|c|c|c|c|c|}
\hline$r \backslash n$ & 1 & 2 & 3 & 4 & 5 & 6 & 7 & 8 & 9 & 10 \\
\hline 1 & 0.894 & 0.561 & 0.37 & 0.247 & 0.164 & 0.107 & 0.069 & 0.044 & 0.028 & 0.017 \\
\hline 2 & & 0.544 & 0.301 & 0.211 & 0.145 & 0.097 & 0.064 & 0.041 & 0.026 & 0.016 \\
\hline 3 & & & 0.242 & 0.177 & 0.126 & 0.086 & 0.058 & 0.038 & 0.024 & 0.015 \\
\hline 4 & & & & 0.145 & 0.106 & 0.075 & 0.052 & 0.034 & 0.022 & 0.014 \\
\hline 5 & & & & & 0.088 & 0.064 & 0.045 & 0.031 & 0.021 & 0.013 \\
\hline 6 & & & & & & 0.053 & 0.038 & 0.027 & 0.018 & 0.012 \\
\hline 7 & & & & & & & 0.032 & 0.023 & 0.016 & 0.011 \\
\hline 8 & & & & & & & & 0.019 & 0.014 & 0.01 \\
\hline 9 & & & & & & & & & 0.011 & 0.008 \\
\hline 10 & & & & & & & & & & 0.007 \\
\hline
\end{tabular}

\section{Restricted families of distributions}

From the numerical results presented in the previous section we see that the smallest upper bias is obtained for $r=1$ i.e. if we consider the mean $S_{n}^{(k)}$ of $k$ th record values. In this section we improve the upper bounds on $E S_{n}^{(k)}$ obtained for general distributions considering restricted classes of distributions: DD and DFR.

In other words we only treat the cases when $W=U$ or $W=V$ in the notation of Sect. 2, but we might generalize it to the case of generalized Pareto distribution $W_{\alpha}$, with arbitrary $\alpha>-1 / 2$ (see Bieniek 2008a). First we need to solve another projection problem.

\subsection{Projection problem}

The projection $P_{W} h$ can be determined for $h \in \mathscr{L}_{W}^{2}$ satisfying the following set of conditions: 
(A) $h:[a, d] \mapsto \mathbb{R}$ is nonnegative, bounded, twice differentiable, $h(a) \geq 0$ and there exist $b$ and $c$ such that $a \leq b<c \leq d$ and $h$ is increasing and convex on $(a, b)$, increasing and concave on $(b, c)$, and decreasing on $(c, d)$. Also we assume that $\int_{a}^{d} h(x) w(x) \mathrm{d} x=1$.

For $W=U$ and $k \geq 2$ the functions $h_{n}^{(k)}$ satisfy (A) by Theorem 2 and for $W=V$ and $k \geq 2$ the functions $\hat{h}_{n}^{(k)}=h_{n}^{(k)} V$ satisfy (A) by Lemma 4 below in Sect. 5.3. Note that the restriction to $r=1$ allows to avoid projecting functions $g_{r, n}^{(k)}$ some of which do not satisfy (A) (see Theorem 3). For instance the functions $g_{2,30}^{(10)}$ and $g_{5,30}^{(20)}$ do not satisfy (A).

The solution to this problem follows the solution to problem of Danielak (2003) who considered the case when $h(a)=0$. First we need to describe the shape of the projection and then we determine its exact parameters.

Lemma 1 Let $\mathscr{C}_{W}^{*} \subset \mathscr{C}_{W}$ be the class of functions $g^{*}:[a, d) \rightarrow \mathbb{R}$ such that

$$
g^{*}(x)= \begin{cases}h(x), & \text { if } a \leq x \leq y, \\ h(y)+\alpha(x-y), & \text { if } x>y,\end{cases}
$$

for some $y \in[a, b]$ and $\alpha \geq h^{\prime}(y)$, or

$$
g^{*}(x)=h(y)+\alpha(x-y),
$$

for some $\alpha \geq 0$ and $a \leq y \leq d$. Then for every $g \in \mathscr{C}_{W}$ there exists $g^{*} \in \mathscr{C}_{W}^{*}$ such that

$$
\|h-g\| \geq\left\|h-g^{*}\right\| .
$$

Proof Assume that $h(a)>0$, and let $\theta \in(c, d)$ be the unique point on the decrease interval of $h$ such that $h(\theta)=h(a)$. If there is no such point then the results of Danielak (2003) are applicable after simple shift.

It suffices to consider only functions $g$ for which $0<g(a)<h(a)$ and two cases:

(i) $g(\theta) \leq h(\theta)$;

(ii) $g(b)<h(\theta)<g(\theta)$.

In the case (i) the functions $g$ and $h$ cross each other at the point $\delta \in(\theta, d)$. Then the constant function equal to $h(\delta)$ is closer to $h$ than $g$ in $\mathscr{L}_{W}^{2}$ norm.

In the case (ii) $g$ and $h$ cross at $\Delta \in(b, \theta)$. Let $l_{\delta \Delta}$ be the straight line passing through the point $(\Delta, g(\Delta))$ and tangent to $h$ at some $\delta \in(a, b)$. Then if

$$
g^{*}(x)= \begin{cases}h(x), & \text { if } a \leq x \leq \delta \\ l_{\delta \Delta}(x), & \text { if } \delta<x<d\end{cases}
$$

then $g^{*}$ is closer to $h$ than $g$.

The proof in the remaining cases follows exactly the proof of Lemma 3 of Danielak (2003). 
The rest of the solution of this projection problem is the same as those of Danielak (2003). Namely, for $h$ satisfying (A) we define

$$
\alpha_{*}(y)=\frac{\int_{y}^{d}(x-y)(h(x)-h(y)) w(x) \mathrm{d} x}{\int_{y}^{d}(x-y)^{2} w(x) \mathrm{d} x} .
$$

Then the shape of $P_{W} h$ depends on the behavior of functions $K_{W}$ and $L_{W}$, where

$$
K_{W}(y)=\alpha_{*}(y)-h^{\prime}(y),
$$

and

$$
L_{W}(y)=\int_{y}^{d}\left[h(x)-h(y)-\alpha_{*}(y)(x-y)\right] w(x) \mathrm{d} x,
$$

defined for $y \in[a, b]$.

Proposition 1 Let $\mathscr{K}=\left\{a \leq y \leq b: K_{W}(y) \geq 0\right.$ and $\left.L_{W}(y)=0\right\}$. If $\mathscr{K} \neq \varnothing$ and $y^{*}=\sup \mathscr{K}$, then

$$
P_{W} h(x)= \begin{cases}h(x), & \text { for } x \leq y^{*} \\ h\left(y^{*}\right)+\alpha_{*}\left(y^{*}\right)\left(x-y^{*}\right), & \text { for } x>y^{*}\end{cases}
$$

Otherwise $(\mathscr{K}=\varnothing)$,

$$
P_{W} h(x)=\max (\bar{\alpha}, 0)(x-a)+\min (\bar{\beta}, 1),
$$

where

$$
\bar{\alpha}=\frac{\int_{a}^{d}(x-a) h(x) w(x) \mathrm{d} x-\int_{a}^{d}(x-a) w(x) \mathrm{d} x}{\int_{a}^{d}(x-a)^{2} w(x) \mathrm{d} x-\left[\int_{a}^{d}(x-a) w(x) \mathrm{d} x\right]^{2}}
$$

and

$$
\bar{\beta}=\frac{\int_{a}^{d}(x-a)^{2} w(x) \mathrm{d} x-\int_{a}^{d}(x-a) h(x) w(x) \mathrm{d} x \int_{a}^{d}(x-a) w(x) \mathrm{d} x}{\int_{a}^{d}(x-a)^{2} w(x) \mathrm{d} x-\left[\int_{a}^{d}(x-a) w(x) \mathrm{d} x\right]^{2}} .
$$

Remark 3 If the projection is linear function of the form (8) then $\bar{\beta} \geq 0$. For if $\bar{\beta}<0$, then $\bar{\alpha}>0$, and (8) would be negative in a neighbourhood of $a$. But then the function $[\bar{\alpha}(x-a)+\bar{\beta}]_{+}$would be a better approximation of $h$ than (8), which is a contradiction.

The following simplified version of Lemma 4 of Gajek and Rychlik (1998) is very useful when we study the function $L_{W}$.

Lemma 2 If $\mathscr{K}^{+}=\left\{y \in(a, b]: K_{W}(y)>0\right\}=(a, v)$ and $L_{W}$ has a finite number of zeros, then $L_{W}$ is either positive or negative or it changes the sign from - to + in $\mathscr{K}^{+}$. 


\subsection{Distributions with decreasing density}

Now we consider the case $W=U$. Then $h_{n}^{(k)}$ satisfies (A) for all $n, k \geq 2$. First we calculate the functions $\alpha_{*}(y), K_{U}$ and $L_{U}$ and the parameters $\bar{\alpha}$ and $\bar{\beta}$. We make use of identities

$$
\int_{y}^{1} f_{n}^{(k)}(x) \mathrm{d} x=\frac{1-y}{k} \sum_{i=1}^{n} f_{i}^{(k)}(y)
$$

and

$$
\int_{y}^{1}(1-x) f_{n}^{(k)}(x) \mathrm{d} x=\frac{(1-y)^{2}}{k} \sum_{i=1}^{n}\left(\frac{k}{k+1}\right)^{n-i+1} f_{i}^{(k)}(y) .
$$

which can be checked by direct computations. They are also special cases of Eqs. (12) and (13) of Bieniek (2008b). We have

$$
\alpha_{*}(y)=\frac{3}{(1-y)^{3}}\left[(1-y) \int_{y}^{1} h_{n}^{(k)}(x) \mathrm{d} x-\int_{y}^{1}(1-x) h_{n}^{(k)}(x) \mathrm{d} x-\frac{1}{2} h_{n}^{(k)}(y)(1-y)^{2}\right] .
$$

By using (9)

$$
\int_{y}^{1} h_{n}^{(k)}(x) \mathrm{d} x=\frac{1-y}{n k} \sum_{i=1}^{n}(n-i+1) f_{i}^{(k)}(y)
$$

and by (10)

$$
\int_{y}^{1}(1-x) h_{n}^{(k)}(x) \mathrm{d} x=\frac{(1-y)^{2}}{n} \sum_{i=1}^{n}\left[1-\left(\frac{k}{k+1}\right)^{n-i+1}\right] f_{i}^{(k)}(y) .
$$

Therefore by (11)

$$
\alpha_{*}(y)=\frac{3}{n(1-y)} \sum_{i=1}^{n}\left[\frac{n-i+1}{k}+\left(\frac{k}{k+1}\right)^{n-i+1}-\frac{3}{2}\right] f_{i}^{(k)}(y)
$$

After detailed computations this gives

$$
K_{U}(y)=\frac{1}{n(1-y)} \sum_{i=1}^{n} \alpha_{i} f_{i}^{(k)}(y)
$$

where

$$
\begin{aligned}
\alpha_{i} & =\frac{3(n-i+1)}{k}+3\left(\frac{k}{k+1}\right)^{n-i+1}-\frac{11}{2}, \quad 1 \leq i<n, \\
\alpha_{n} & =\frac{(k-1)(k-2)(2 k+3)}{2 k(k+1)},
\end{aligned}
$$


and

$$
L_{U}(y)=\frac{1-y}{n} \sum_{i=1}^{n} \beta_{i} f_{i}^{(k)}(y)
$$

where for $1 \leq i \leq n$

$$
\beta_{i}=\frac{5}{4}-\frac{n-i+1}{2 k}-\frac{3}{2}\left(\frac{k}{k+1}\right)^{n-i+1} .
$$

To calculate the values of $\bar{\alpha}$ and $\bar{\beta}$ it suffices to know the value of the integral

$$
\begin{aligned}
\int_{0}^{1} x h_{n}^{(k)}(x) \mathrm{d} x & =1-\int_{0}^{1}(1-x) h_{n}^{(k)}(x) \mathrm{d} x \\
& =1-\frac{1}{n} \sum_{i=1}^{n}\left[1-\left(\frac{k}{k+1}\right)^{n-i+1}\right] f_{i}^{(k)}(0) \\
& =1-\frac{k}{n}\left[1-\left(\frac{k}{k+1}\right)^{n}\right] .
\end{aligned}
$$

We have used (12) here. Therefore

$$
\bar{\alpha}=12 \int_{0}^{1} x h_{n}^{(k)}(x) \mathrm{d} x-6=6-\frac{12 k}{n}\left[1-\left(\frac{k}{k+1}\right)^{n}\right],
$$

and

$$
\bar{\beta}=4-6 \int_{0}^{1} x h_{n}^{(k)}(x) \mathrm{d} x=\frac{6 k}{n}\left[1-\left(\frac{k}{k+1}\right)^{n}\right]-2 .
$$

We also need the following analytical lemma which will be helpul in statement and the proof of the next theorem. The proof of the lemma is given in the "Appendix".

Lemma 3 Let $n, k \geq 1$ be positive integers. Then

(a) if $1 \leq n \leq k$, then

$$
\frac{n}{k}+2\left(\frac{k}{k+1}\right)^{n} \leq 2 \text { and } \frac{n}{k}+\left(\frac{k}{k+1}\right)^{n} \leq \frac{11}{6}
$$

(b) if $n \geq k \geq 1$

$$
\frac{n}{k}+3\left(\frac{k}{k+1}\right)^{n}>\frac{5}{2} \Longrightarrow \frac{n}{k}+2\left(\frac{k}{k+1}\right)^{n}>2
$$

(c) for all $n, k \geq 1$

$$
\frac{n}{k}+3\left(\frac{k}{k+1}\right)^{n}>3 \Longrightarrow \frac{n}{k}+2\left(\frac{k}{k+1}\right)^{n}>2
$$

(d) if $n \geq k \geq 1$

$$
\frac{n}{k}+3\left(\frac{k}{k+1}\right)^{n}>3 \Longrightarrow \frac{n}{k}+\left(\frac{k}{k+1}\right)^{n}>\frac{11}{6} .
$$

In our notation the statement of Lemma 3 can be rephrased as follows: 
(a) if $n \leq k$ then $\bar{\alpha} \leq 0$ and $\alpha_{1} \leq 0$;

(b) if $n \geq k$ then $\bar{\alpha} \leq 0$ implies $\beta_{1} \geq 0$;

(c) if $\bar{\beta}<0$ then $\bar{\alpha}>0$;

(d) if $n \geq k$ then $\bar{\beta}<0$ implies $\alpha_{1}>0$.

Now we may state our main result on bounds on expectations of $S_{n}^{(k)}$ for distributions with decreasing density. Note that for $k=1$ by Theorem 2(a) the function $h_{n}^{(1)}$ is convex increasing. So $P_{U} h_{n}^{(1)}=h_{n}^{(1)}$ and the upper bounds on $E h_{n}^{(1)}=E T_{1, n}^{(1)}$ given in Theorem 4 are optimal in DD family as well. Therefore in the rest of this subsection we assume that $k \geq 2$.

Theorem 5 Fix any $F \in D D$ with finite mean $\mu$ and variance $\sigma^{2}$ and $n, k \geq 2$.

$$
\begin{aligned}
& \text { If } \frac{n}{k}+2\left(\frac{k}{k+1}\right)^{n} \leq 2 \text {, then } E S_{n}^{(k)} \leq \mu . \\
& \text { If } \frac{n}{k}+2\left(\frac{k}{k+1}\right)^{n}>2 \text { and }
\end{aligned}
$$$$
\text { either } \frac{n}{k}+\left(\frac{k}{k+1}\right)^{n} \leq \frac{11}{6} \text { or } \frac{n}{k}+3\left(\frac{k}{k+1}\right)^{n} \leq \frac{5}{2} \quad \text { or } \quad L_{U}(v) \leq 0
$$

where $v$ is the smallest solution to $K_{U}(v)=0$ in $(0,1)$, then

$$
\frac{E S_{n}^{(k)}-\mu}{\sigma} \leq C_{U}(n, k):=\sqrt{3}(1-2 \bar{C}),
$$

where

$$
\bar{C}=\bar{C}(n, k)=\frac{k}{n}\left(1-\left(\frac{k}{k+1}\right)^{n}\right),
$$

and the bound is attained for uniform distribution on $(\mu-\sigma \sqrt{3}, \mu+\sigma \sqrt{3})$.

Otherwise, i.e. if $\frac{n}{k}+2\left(\frac{k}{k+1}\right)^{n}>2$ and

$$
\frac{n}{k}+\left(\frac{k}{k+1}\right)^{n}>\frac{11}{6} \text { and } \frac{n}{k}+3\left(\frac{k}{k+1}\right)^{n}>\frac{5}{2} \text { and } L_{U}(v)>0
$$

then

$$
\frac{E S_{n}^{(k)}-\mu}{\sigma} \leq C=C_{U}(n, k)
$$

where

$$
\begin{aligned}
C^{2}= & \int_{0}^{y^{*}}\left(h_{n}^{(k)}(x)\right)^{2} \mathrm{~d} x+\left(h_{n}^{(k)}\left(y^{*}\right)\right)^{2}\left(1-y^{*}\right) \\
& +\left(\alpha_{*}\left(y^{*}\right)\right)^{2} \frac{\left(1-y^{*}\right)^{3}}{3}+h_{n}^{(k)}\left(y^{*}\right) \alpha_{*}\left(y^{*}\right)\left(1-y^{*}\right)^{2}-1,
\end{aligned}
$$

and $y^{*}$ is the unique solution to $L_{U}(y)=0$ in $(0, v)$. The bound is attained if 


$$
F(x)= \begin{cases}0, & \text { if } \frac{x-\mu}{\sigma}<\frac{k-n}{n C}, \\ \left(h_{n}^{(k)}\right)^{-1}\left(C \frac{x-\mu}{\sigma}+1\right), & \text { if } \frac{k-n}{n C} \leq \frac{x-\mu}{\sigma}<A, \\ y^{*}+\frac{C(x-\mu)}{\sigma \alpha_{*}\left(y^{*}\right)}+\frac{1-h_{n}^{(k)}\left(y^{*}\right)}{\alpha_{*}\left(y^{*}\right)}, & \text { if } A \leq \frac{x-\mu}{\sigma}<B, \\ 1, & \text { if } \frac{x-\mu}{\sigma} \geq B,\end{cases}
$$

with

$$
A=\frac{h_{n}^{(k)}\left(y^{*}\right)-1}{C}, \quad B=\frac{h_{n}^{(k)}\left(y^{*}\right)+\left(1-y^{*}\right) \alpha_{*}\left(y^{*}\right)-1}{C} .
$$

Proof First we note that the sequence $\alpha_{1}, \ldots, \alpha_{n-1}$ is strictly decreasing to $\alpha_{n-1}<0$ for $k=2,3, \ldots$ and $\alpha_{n} \geq 0$, and $K_{U}(b)<0$.

If $\alpha_{1} \leq 0$ then by Theorem 1 the function $K_{U}$ is $-+(-$ if $k=2)$ on $(0,1)$, so it is negative on $(0, b)$ and $\mathscr{K}=\varnothing$, and $P_{U} h_{n}^{(k)}$ is a linear function. If $\alpha_{1}>0$, then $K_{U}$ is either +-+ or $+(+-$ if $k=2)$ on $(0,1)$. But $K_{U}(b)<0$, so $K_{U}$ cannot be positive, so in both cases it is +- on $(0, b)$ and $\mathscr{K}^{+}=(0, v)$ where $v$ is the smallest solution to $K_{U}(v)=0$ on $(0,1)$, and to determine $P_{U} h_{n}^{(k)}$ we need to study the behaviour of the function $L_{U}$.

Now $\beta_{n}=0$ if $k=2$ and $\beta_{n}<0$ for $k \geq 3$, and $\beta_{n-1}>0$ for $k \geq 2$, and routine calculations show that the sequence $\beta_{1}, \ldots, \beta_{n}$ is either decreasing or first increasing and then decreasing. Therefore $L_{U}$ is either -+- or -+ or +- or + or - on $(0,1)$ depending on the value of $k$ and the sign of $\beta_{1}$.

Namely if $\beta_{1} \geq 0$, then by Lemma 2 the function $L_{U}$ has to be positive on $(0, v)$ and again $\mathscr{K}=\varnothing$ and $P_{U} h_{n}^{(k)}$ is a strictly linear function. If $\beta_{1}<0$ then using Lemma 2 again, $L_{U}$ is either -+ of - on $(0, v)$ and the shape of $P_{U} h_{n}^{(k)}$ depends on whether $L_{U}(v) \leq 0$ or $L_{U}(v)>0$. In the former case $L_{U}$ is negative on $(0, v)$ and again $\mathscr{K}=\varnothing$ and the projection is linear. In the latter case $L_{U}$ has unique zero $y^{*}$ in $(0,1)$ and the projection is

$$
P_{U} h_{n}^{(k)}(x)= \begin{cases}h_{n}^{(k)}(x), & \text { for } 0 \leq x \leq y^{*} \\ h_{n}^{(k)}\left(y^{*}\right)+\alpha_{*}\left(y^{*}\right)\left(x-y^{*}\right), & \text { for } y^{*}<x \leq 1\end{cases}
$$

Summing up, if

$$
\alpha_{1}>0, \quad \beta_{1}<0 \text { and } L_{U}(v)>0
$$

then the projection is of the above form. If

$$
\alpha_{1} \leq 0 \text { or } \beta_{1} \geq 0 \text { or } L_{U}(v) \leq 0
$$

then the projection is the linear function of the form

$$
P_{U} h_{n}^{(k)}(x)=(6-12 \bar{C}) x+(6 \bar{C}-2) .
$$

If additionally $\bar{\alpha} \leq 0$, then the projection is constant equal to 1 .

We now analyze when (17) or (18) holds depending on $n$ and $k$. First we assume that $\frac{n}{k}+2\left(\frac{k}{k+1}\right)^{n} \leq 2$ i.e. $\bar{\alpha} \leq 0$. If $n \leq k$, then Lemma 3(a) implies that $\alpha_{1} \leq 0$, 
and if $n>k$ then Lemma 3(b) implies that $\beta_{1} \geq 0$. In both cases (18) holds and since $\bar{\alpha} \leq 0$ we get that $P_{U} \hat{h}_{n}^{(k)}=1$. If we assume that $\frac{n}{k}+2\left(\frac{k}{k+1}\right)^{n}>2$, i.e. $\bar{\alpha}>0$ then (18) is equivalent to (13), and (17) is equivalent to (15). Now the detailed statements of the theorem follow after calculations of the norms of the projections and the forms of distributions for which equalities hold follow from (5).

We conclude this subsection with a short discussion on the conditions of Theorem 5.

Remark 4 1. If $n \leq k$ then by Lemma 3(a) we get $n / k+\left(\frac{k}{k+1}\right)^{n} \leq 2$, so by Theorem 5 we have $E S_{n}^{(k)} \leq \mu$. This agrees with the thesis of Theorem 4 on bounds for general distributions, since bounds in DD family cannot be greater than general bounds.

2. If instead of the second inequality in (15) we assume that

$$
\frac{n}{k}+3\left(\frac{k}{k+1}\right)^{n}>3
$$

then obviously $\beta_{1}<0$ and by Lemma 3(c) it follows that $\bar{\alpha}>0$, so $n \geq k$ and by Lemma 3(d) we get $\alpha_{1}>0$. Moreover $L_{U}(v)>0$. Indeed, if $L_{U}(v) \leq 0$, then the projection would be a linear function with $\bar{\beta}<0$, which is impossible by Remark 3. So (19) suffices to claim that (15) holds.

3. The only values of $n$ for which

$$
\frac{n}{k}+\left(\frac{k}{k+1}\right)^{n}>\frac{11}{6} \text { and } \frac{5}{2}<\frac{n}{k}+3\left(\frac{k}{k+1}\right)^{n} \leq 3
$$

are

$-n=3,4$ if $k=2$;

$-n=6,7,8$ if $k=3$;

$-n=8,9,10$ if $k=4$;

$-n=11,12,13$ if $k=5$.

Moreover numerical calculations show that if $k=3$ and $n=6$ then $\alpha_{1}>0$, $\beta_{1}<0$ and $L_{U}(v)<0$, while if $k=3$ and $n=7$ then $\alpha_{1}>0, \beta_{1}<0$ and $L_{U}(v)>0$, so both possibilities do occur. This is contrary to order statistic case when the respective $\beta_{1}<0$ excludes the case of linear projection.

\subsection{Distributions with decreasing failure rate}

Now we consider the case $W=V$ and $\hat{h}_{n}^{(k)}=h_{n}^{(k)} V$. First we determine when the functions $\hat{h}_{n}^{(k)}$ satisfy the conditions (A). We use the identities

$$
\left(\hat{h}_{n}^{(k)}\right)^{\prime}(x)=\frac{1}{n}\left(\sum_{i=1}^{n-1} \hat{f}_{i}^{(k)}(x)-(k-1) \hat{f}_{n}^{(k)}(x)\right)
$$


and

$$
\left(\hat{h}_{n}^{(k)}\right)^{\prime \prime}(x)=\frac{1}{n}\left(\sum_{i=1}^{n-2} \hat{f}_{i}^{(k)}(x)+\left(-k^{2}+1\right) \hat{f}_{n-1}^{(k)}(x)+(k-1)^{2} \hat{f}_{n}^{(k)}(x)\right) .
$$

Analyzing the sign changes of the coefficients of the above expansions we prove the following lemma.

Lemma 4 (a) Let $k=1$. The function $\hat{h}_{2}^{(1)}$ is linear increasing, and the functions $\hat{h}_{n}^{(1)}, n \geq 3$, are convex increasing.

(b) Let $k \geq 2$. The functions $\hat{h}_{n}^{(k)}, n \geq 2$, are increasing from $k / n$, and then decreasing to 0. Moreover:

(i) the functions $\hat{h}_{2}^{(k)}, k \geq 2$, are concave-convex;

(ii) the functions $\hat{h}_{n}^{(k)}, n \geq 3$, are convex-concave-convex.

Therefore the functions $\hat{h}_{n}^{(k)}$ satisfy the conditions (A) for all $k \geq 2$ and $n \geq 2$.

Next we need to calculate the functions $\alpha_{*}, K_{V}, L_{V}$ and the parameters $\bar{\alpha}$ and $\bar{\beta}$. We use the identities

$$
\int_{y}^{\infty} \hat{f}_{n}^{(k)}(x) \mathrm{e}^{-x} \mathrm{~d} x=\frac{\mathrm{e}^{-y}}{k} \sum_{i=1}^{n} \hat{f}_{i}^{(k)}(y)
$$

and

$$
\int_{y}^{\infty}(x-y) \hat{f}_{n}^{(k)}(x) \mathrm{e}^{-x} \mathrm{~d} x=\frac{\mathrm{e}^{-y}}{k^{2}} \sum_{i=1}^{n}(n-i+1) \hat{f}_{i}^{(k)}(y)
$$

(see e.g. Bieniek 2008a, “Appendix”). These relations easily imply

$$
\int_{y}^{\infty} \hat{h}_{n}^{(k)}(x) \mathrm{e}^{-x} \mathrm{~d} x=\frac{\mathrm{e}^{-y}}{n k} \sum_{i=1}^{n}(n-i+1) \hat{f}_{i}^{(k)}(y)
$$

and

$$
\int_{y}^{\infty}(x-y) \hat{h}_{n}^{(k)}(x) \mathrm{e}^{-x} \mathrm{~d} x=\frac{\mathrm{e}^{-y}}{2 n k^{2}} \sum_{i=1}^{n}(n-i+1)(n-i+2) \hat{f}_{n}^{(k)}(y) .
$$

Now for $W=V$ we have

$$
\begin{aligned}
\alpha_{*}(y) & =\frac{\mathrm{e}^{y}}{2}\left[\int_{y}^{\infty}(x-y) \hat{h}_{n}^{(k)}(x) \mathrm{e}^{-x} \mathrm{~d} x-\hat{h}_{n}^{(k)}(y) \mathrm{e}^{-y}\right] \\
& =\frac{1}{2 n} \sum_{i=1}^{n}\left[\frac{(n-i+1)(n-i+2)}{2 k^{2}}-1\right] \hat{f}_{i}^{(k)}(y),
\end{aligned}
$$


which gives

$$
K_{V}(y)=\frac{1}{n} \sum_{i=1}^{n} \gamma_{i} \hat{f}_{i}^{(k)}(y)
$$

where

$$
\begin{aligned}
& \gamma_{i}=\frac{(n-i+1)(n-i+2)}{4 k^{2}}-\frac{3}{2}, \quad 1 \leq i<n, \\
& \gamma_{n}=\frac{(k-1)^{2}(2 k+1)}{2 k^{2}} .
\end{aligned}
$$

Moreover,

$$
L_{V}(y)=\frac{\mathrm{e}^{-y}}{n} \sum_{i=1}^{n} \delta_{i} \hat{f}_{i}^{(k)}(y)
$$

where

$$
\delta_{i}=\frac{n-i+1}{k}\left(1-\frac{n-i+2}{4 k}-\frac{k}{2(n-i+1)}\right), \quad 1 \leq i \leq n .
$$

To compute $\bar{\alpha}$ and $\bar{\beta}$ it suffices to calulate the value of the integral

$$
\int_{0}^{\infty} x \hat{h}_{n}^{(k)}(x) \mathrm{e}^{-x} \mathrm{~d} x=\frac{1}{2 n k^{2}} \sum_{i=1}^{n}(n-i+1)(n-i+2) \hat{f}_{i}^{(k)}(0)=\frac{n+1}{2 k}
$$

Therefore

$$
\bar{\alpha}=\int_{0}^{\infty} x \hat{h}_{n}^{(k)}(x) \mathrm{e}^{-x} \mathrm{~d} x-1=\frac{n+1}{2 k}-1,
$$

and

$$
\bar{\beta}=2-\int_{0}^{\infty} x \hat{h}_{n}^{(k)}(x) \mathrm{e}^{-x} \mathrm{~d} x=2-\frac{n+1}{2 k} .
$$

Now we may present the main result of this subsection on bounds on $E S_{n}^{(k)}$ valid for DFR distributions. In DFR case the conditions on $n$ and $k$ have more explicit form. We exclude the case $k=1$ for the same reason as in DD case. The upper bounds on $E S_{n}^{(1)}$ for general distributions are attained by DFR distributions and hence are optimal in the DFR family as well.

Theorem 6 Fix any $F \in D F R$ with finite mean $\mu$ and variance $\sigma^{2}$ and $n, k \geq 2$.

$$
\begin{aligned}
& \text { If } 2 \leq n \leq 2 k-1 \text {, then } E S_{n}^{(k)} \leq \mu \text {. } \\
& \text { If } n>2 k-1 \text {, and }
\end{aligned}
$$

$$
\text { either } n \leq \frac{1}{2}\left(4 k-1+\sqrt{(4 k-1)^{2}-8 k^{2}}\right) \quad \text { or } \quad L_{V}(v) \leq 0 \text {, }
$$


where $v$ is the smallest positive solution to $K_{V}(v)=0$, then

$$
\frac{E S_{n}^{(k)}-\mu}{\sigma} \leq C_{V}(n, k):=\frac{n+1}{2 k}-1
$$

and the bound is attained for the exponential distribution of the form

$$
F(x)=1-\exp \left(-\frac{x-\mu}{\sigma}+1\right), \quad x \geq \mu
$$

If

$$
n>\frac{1}{2}\left(4 k-1+\sqrt{(4 k-1)^{2}-8 k^{2}}\right) \text { and } L_{V}(v)>0
$$

then

$$
\frac{E S_{n}^{(k)}-\mu}{\sigma} \leq C=C_{V}(n, k)
$$

where

$$
C^{2}=\int_{0}^{y^{*}}\left(\hat{h}_{n}^{(k)}(x)\right)^{2} \mathrm{e}^{-x} \mathrm{~d} x+\mathrm{e}^{-y^{*}}\left[\left(\hat{h}_{n}^{(k)}\left(y^{*}\right)\right)^{2}+2 \hat{h}_{n}^{(k)}\left(y^{*}\right) \alpha_{*}\left(y^{*}\right)+2\left(\alpha_{*}\left(y^{*}\right)\right)^{2}-1\right]
$$

and $y^{*}$ is the unique solution to $L_{V}(v)=0$ in $(0, v)$. The bound is attained if

$$
F(x)= \begin{cases}0, & \text { if } \frac{x-\mu}{\sigma}<\frac{k-n}{n C}, \\ \left(h_{n}^{(k)}\right)^{-1}\left(C \frac{x-\mu}{\sigma}+1\right), & \text { if } \frac{k-n}{n C} \leq \frac{x-\mu}{\sigma}<\frac{\hat{h}_{n}^{(k)}\left(y^{*}\right)-1}{C}, \\ V\left(y^{*}+\frac{C(x-\mu)}{\sigma \alpha_{*}\left(y^{*}\right)}+\frac{1-\hat{h}_{n}^{(k)}\left(y^{*}\right)}{\alpha_{*}\left(y^{*}\right)}\right), & \text { if } \frac{x-\mu}{\sigma} \geq \frac{\hat{h}_{n}^{(k)}\left(y^{*}\right)-1}{C} .\end{cases}
$$

Proof The proof is similar to the proof of Theorem 5 so we only describe most essential steps here.

First note that the sequence $\gamma_{1}, \ldots, \gamma_{n-1}$ is decreasing to $\gamma_{n-1}=\frac{3}{2}\left(\frac{1}{k^{2}}-1\right)<0$ and $\gamma_{n}>0$ (for $k \geq 2$ ). Therefore if $\gamma_{1} \leq 0$, then $K_{V}$ is -+ on $(0, \infty)$, and since $K_{V}(b)<0$, the function $K_{V}$ is negative on $(0, b)$, so $\mathscr{K}=\varnothing$ and the projection is linear.

If $\gamma_{1}>0$ then $K_{V}$ is +-+ on $(0, \infty)$ (it cannot be + since $K_{V}(b)<0$ ) and $\mathscr{K}^{+}=(0, v)$ where $v$ is the smallest positive zero of $K_{V}$. To determine the shape of $P_{V} \hat{h}_{n}^{(k)}$ we need to analyze $L_{V}$.

Note that the sequence $\delta_{1}, \ldots, \delta_{n}$ is either decreasing or increasing-decreasing to $\delta_{n}=-\frac{1}{2 k}(k-1)^{2}<0$ (for $k \geq 2$ ). If $\delta_{1} \geq 0$ then $L_{V}$ is +- on $(0, \infty)$ and therefore it is positive on $(0, v)$ and again $\mathscr{K}=\varnothing$ and the projection is linear. If $\delta_{1}<0$ then 
$L_{U}$ is either - or -+- on $(0, \infty)$ so by Lemma 2 it is either - or -+ on $(0, v)$. If $L_{V}(v) \leq 0$ then $\mathscr{K}=\varnothing$ and $P_{V} \hat{h}_{n}^{(k)}$ is linear and if $L_{V}(v)>0$ then the projection is

$$
P_{V} \hat{h}_{n}^{(k)}(x)= \begin{cases}\hat{h}_{n}^{(k)}(x), & \text { for } 0 \leq x \leq y^{*} \\ \hat{h}_{n}^{(k)}\left(y^{*}\right)+\alpha_{*}\left(y^{*}\right)\left(x-y^{*}\right), & \text { for } x>y^{*}\end{cases}
$$

where $y^{*}$ is the unique zero of $L_{V}$ in $(0, v)$.

Summing up if $\gamma_{1}>0$ and $\delta_{1}<0$ and $L_{V}(v)>0$ then $P_{V} \hat{h}_{n}^{(k)}$ is of the form (23) and otherwise it is linear, and if $\bar{\alpha} \leq 0$ it is constant equal to 1 . To describe the conditions on the signs of $\bar{\alpha}, \gamma_{1}$ and $\delta_{1}$ in terms of $n$ and $k$, we note that for $n \geq 1$

$$
\begin{aligned}
& \bar{\alpha} \leq 0 \Leftrightarrow n \leq n_{\alpha}=2 k-1, \\
& \gamma_{1}>0 \Leftrightarrow n>n_{\gamma}=\frac{1}{2}\left(\sqrt{24 k^{2}+1}-1\right), \\
& \delta_{1} \geq 0 \Leftrightarrow n_{\delta 1} \leq n \leq n_{\delta 2}, \\
& \delta_{1}<0 \Leftrightarrow \text { either } 1 \leq n<n_{\delta 1} \text { or } n>n_{\delta 2},
\end{aligned}
$$

where

$$
n_{\delta 1,2}=\frac{1}{2}\left(4 k-1 \mp \sqrt{(4 k-1)^{2}-8 k^{2}}\right) .
$$

We can check that for $k \geq 1$ we have $0<n_{\delta 1}<n_{\alpha}<n_{\gamma}<n_{\delta 2}$. Therefore for $n \leq n_{\delta 1}$ we have $\gamma_{1} \leq 0$, so the projection is linear and since $\bar{\alpha} \leq 0$, it is constant equal to 1 . For $n_{\delta 1}<n \leq n_{\alpha}$ we have $\delta_{1} \geq 0$, so the projection is linear, and since $\bar{\alpha} \leq 0$, it is again constant. For $n_{\alpha}<n \leq n_{\delta 2}$ we have and $\delta_{1} \geq 0$, but $\bar{\alpha}>0$, so the projection is strictly increasing linear function. Finally for $n>n_{\delta 2}$ we have $\gamma_{1}>0$ and $\delta_{1}<0$ and the verification of the sign of $L_{V}(v)$ is nedeed.

Remark 5 If $n>4 k-1$ then the condition (21) holds. Indeed, if $L_{V}(v)$ was negative then the projection $P_{V} \hat{h}_{n}^{(k)}$ would be linear function with $\bar{\beta}<0$ which is impossible by Remark 3. Also similar to DD case we can find vaues of $n \leq 4 k-1$ for which $\gamma_{1}>0, \delta_{1}<0$ but $L_{V}(v) \leq 0$ so $P_{V} \hat{h}_{n}^{(k)}$ is linear.

\section{Numerical results}

The results of the previous section allow numerical implementation. In Table 5 we compare the lower and upper general bounds $-\underline{B}_{1, n}^{(k)}$ and $\bar{B}_{1, n}^{(k)}$ with bounds in restricted families $C_{U}(n, k)$ and $C_{V}(n, k)$ for $k=2$ and $k=3$ and $1 \leq n \leq 10$. The values of $\underline{B}_{1, n}^{(3)}$ are more accurate values of the numbers in the first row of Table 4 . The values in bold are obtained from the simple formulae (14) and (20), and the remaining positive values of $C_{U}(n, k)$ and $C_{V}(n, k)$ are obtained from the complicated formulae (16) and (22).

Obviously the bounds obtained for DFR case are tighter than in DD case, since the class DFR is narrower than DD. Also we see that the gain by restricting to DD or DFR distributions is smaller than that obtained by looking at $k$ th record values with 
Table 5 Comparision of general and restricted bounds

\begin{tabular}{|c|c|c|c|c|c|c|c|c|}
\hline \multirow[t]{2}{*}{$n$} & \multicolumn{4}{|l|}{$k=2$} & \multicolumn{4}{|l|}{$k=3$} \\
\hline & $-\underline{B}_{1, n}^{(2)}$ & $C_{V}(n, 2)$ & $C_{U}(n, 2)$ & $\bar{B}_{1, n}^{(2)}$ & $-\underline{B}_{1, n}^{(3)}$ & $C_{V}(n, 3)$ & $C_{U}(n, 3)$ & $\bar{B}_{1, n}^{(3)}$ \\
\hline 1 & -0.5774 & 0 & 0 & 0 & -0.8944 & 0 & 0 & 0 \\
\hline 2 & -0.2684 & 0 & 0 & 0 & -0.5604 & 0 & 0 & 0 \\
\hline 3 & -0.2191 & 0 & 0.1069 & 0.154 & -0.3698 & 0 & 0 & 0 \\
\hline 4 & -0.0447 & 0.25 & 0.3433 & 0.353 & -0.2468 & 0 & 0 & 0.0878 \\
\hline 5 & -0.0158 & 0.5 & 0.5589 & 0.562 & -0.1637 & 0 & 0.1468 & 0.2091 \\
\hline 6 & -0.0053 & 0.751 & 0.786 & 0.787 & -0.1073 & 0.1667 & 0.3083 & 0.3369 \\
\hline 7 & -0.0017 & 1.012 & 1.037 & 1.038 & -0.0694 & 0.3333 & 0.4527 & 0.4687 \\
\hline 8 & -0.0005 & 1.307 & 1.325 & 1.326 & -0.0443 & 0.5 & 0.5953 & 0.6057 \\
\hline 9 & -0.0001 & 1.647 & 1.662 & 1.663 & -0.0279 & 0.6667 & 0.7426 & 0.7499 \\
\hline 10 & -0.00007 & 2.052 & 2.064 & 2.065 & -0.0175 & 0.8347 & 0.8983 & 0.9039 \\
\hline
\end{tabular}

$k \geq 2$ instead of ordinary records, and this gain becomes smaller and smaller as $n$ increases. The values of lower bounds in the table show that it is not worth deriving the lower bounds in restricted families, at least for small values of $k$. On the other hand large values of $k$ require large amount of data to observe $k$ th records, so this case is irrelevant from practical point of view.

Acknowledgments The author wishes to thank to anonymous referee whose comments and remarks were very helpful while preparing the final version of the paper.

Open Access This article is distributed under the terms of the Creative Commons Attribution License which permits any use, distribution, and reproduction in any medium, provided the original author(s) and the source are credited.

\section{Appendix: Proof of Lemma 3}

Proof (a) For arbitrarily fixed $k \geq 1$ consider

$$
f_{k}(x)=\frac{x}{k}+2\left(\frac{k}{k+1}\right)^{x}, \quad 0 \leq x \leq k
$$

Then

$$
f_{k}^{\prime \prime}(x)=2\left(\frac{k}{k+1}\right)^{x} \log ^{2}\left(\frac{k}{k+1}\right)>0,
$$

so $f_{k}$ does not have local maxima and maximal value of $f_{k}$ on the interval $[0, k]$ is attained at one of its endpoints. But $f_{k}(0)=2$ and since $\left(\frac{k}{k+1}\right)^{k} \leq \frac{1}{2}$, then

$$
f_{k}(k)=1+2\left(\frac{k}{k+1}\right)^{k} \leq 2
$$




\section{Similarly}

$$
\frac{n}{k}+\left(\frac{k}{k+1}\right)^{n} \leq 1+\frac{1}{2}<\frac{11}{6} .
$$

(b) Assume that $n \geq k>1$. Then

$$
2 \leq\left(1+\frac{1}{k}\right)^{k} \leq\left(1+\frac{1}{k}\right)^{n}
$$

so $\left(\frac{k}{k+1}\right)^{n} \leq \frac{1}{2}$. Therefore, if $\frac{n}{k}+2\left(\frac{k}{k+1}\right)^{n} \leq 2$, then

$$
\frac{n}{k}+3\left(\frac{k}{k+1}\right)^{n} \leq 2+\left(\frac{k}{k+1}\right)^{n} \leq \frac{5}{2} .
$$

(c) If $\frac{n}{k}+3\left(\frac{k}{k+1}\right)^{n}>3$, but $\frac{n}{k}+2\left(\frac{k}{k+1}\right)^{n} \leq 2$, then it easily follows that $\left(\frac{k}{k+1}\right)^{n}>1$ which is a contradiction.

(d) If $\frac{n}{k}+\left(\frac{k}{k+1}\right)^{n} \leq \frac{11}{6}$, then by the proof of (b) it easily follows that $\frac{n}{k}+3\left(\frac{k}{k+1}\right)^{n} \leq$ $\frac{17}{6}<3$.

\section{References}

Bieniek M (2008a) On families of distributions for which optimal bounds on expectations of GOS can be derived. Commun Stat Theory Methods 37:1997-2009

Bieniek M (2008b) Projection bounds on expectations of generalized order statistics from DD and DDA families. J Stat Plan Inference 138:971-981

Danielak K (2003) Sharp upper mean-variance bounds for trimmed means from restricted families. Statistics 37:305-324

Danielak K, Rychlik T (2003) Exact bounds for the bias of trimmed means. Aust NZ J Stat 45:83-96

Dziubdziela W, Kopociński B (1976) Limiting properties of the $k$-th record values. Zastos Mat 15:187-190

Gajek L, Okolewski A (2003) Projection bounds on expectations of record statistics from restricted families. J Stat Plan Inference 110:97-108

Gajek L, Rychlik T (1998) Projection method for moment bounds on order statistics from restricted families. II. Independent case. J Multivar Anal 64:156-182

Goroncy A, Rychlik T (2011) Lower bounds on the expectations of upper record values. J Stat Plan Inference 141:2726-2737

Komornik V (2006) Another short proof of Descartes's rule of signs. Am Math Mon 113:829-830

Moriguti S (1953) A modification of Schwarz's inequality with applications to distributions. Ann Math Stat 24:107-113

Nagaraja HN (1978) On the expected values of record values. Aust J Stat 20:176-182

Raqab MZ (1997) Bounds based on greatest convex minorants for moments of record values. Stat Probab Lett 36:35-41

Raqab MZ, Rychlik T (2002) Sharp bounds for the mean of $k$ th record value. Commun Stat Theory Methods $31: 1927-1937$

Rychlik T (2001) Projecting statistical functionals, Lecture notes in statistics, vol 160. Springer, New York Wang X (2004) A simple proof of Descartes's rule of signs. Am Math Mon 111:525-526 